\title{
Brief communication: Wind-speed-independent actuator disk control for faster annual energy production calculations of wind farms using computational fluid dynamics
}

\author{
Maarten Paul van der Laan ${ }^{1}$, Søren Juhl Andersen ${ }^{2}$, and Pierre-Elouan Réthoré ${ }^{1}$ \\ ${ }^{1}$ Technical University of Denmark, DTU Wind Energy, Risø Campus, \\ Frederiksborgvej 399, 4000 Roskilde, Denmark \\ ${ }^{2}$ Technical University of Denmark, DTU Wind Energy, Lyngby Campus, \\ Anker Engelunds Vej 1, 2800 Lyngby, Denmark \\ Correspondence: Maarten Paul van der Laan (plaa@dtu.dk)
}

Received: 22 May 2019 - Discussion started: 28 May 2019

Revised: 14 September 2019 - Accepted: 6 November 2019 - Published: 2 December 2019

\begin{abstract}
A simple wind-speed-independent actuator disk control method is proposed that can be applied to speed up annual energy production calculations of wind farms using Reynolds-averaged Navier-Stokes simulations. The new control method allows the user to simulate the effect of different wind speeds in one simulation by scaling a calibrated thrust coefficient curve, while keeping the inflow constant. Since the global flow is not changed, only the local flow around the actuator disks need be recalculated from a previous converged result, which reduces the number of required iterations and computational effort by a factor of about 2-3.
\end{abstract}

\section{Introduction}

Wind turbine wakes cause energy losses in wind farms (Barthelmie et al., 2007) and increase blade fatigue loads. The energy losses can be minimized by optimizing the wind farm layout and wind farm control. Fast-engineering wake models (Göçmen et al., 2016) are often used to calculate wake effects in optimization routines; however, their accuracy is not guaranteed, and higher-fidelity wake models are required to validate the annual energy production (AEP) of an optimized wind farm layout. Assessing the AEP for a given wind farm requires on the order of $10^{3}$ computations to account for all wind speeds and wind directions. Reynoldsaveraged Navier-Stokes (RANS) is a relatively fast, yet highfidelity, computational fluid dynamics (CFD) method that can be used for this purpose (van der Laan et al., 2015b), although it is still computationally expensive compared to the traditional engineering approaches.

The wind turbines in RANS are often represented by actuator disks (ADs), where the wind turbine forces are im- plemented as a sink term in the momentum equations. The Reynolds number of the RANS-AD simulations, based on a rotor diameter of $100 \mathrm{~m}$, is on the order of $10^{7}-10^{8}$ for a wind speed range of $4-25 \mathrm{~m} \mathrm{~s}^{-1}$. For these large Reynolds numbers, RANS simulations of a single $\mathrm{AD}$ are Reynolds number independent. In other words, the wake deficit, normalized by the inflow wind speed, does not depend on the inflow wind speed but only depends on the thrust coefficient and atmospheric conditions as ambient turbulence intensity and atmospheric stratification. A proof of the Reynolds number independence of our RANS simulations for a single AD is presented in Appendix A. The Reynolds number independence only holds if the inflow scales by a velocity scale, i.e. the friction velocity. This is true for atmospheric surface layer profiles following Monin-Obukhov similarity theory, where the turbulence length scale is also invariant of the wind speed. For RANS-AD simulations of wind farms, the thrust coefficient varies within the wind farm, which means that one still needs to simulate multiple wind speed cases despite the Reynolds number independence of the flow. 
In previous work (van der Laan et al., 2015a), an AD control method was developed for RANS simulations of interacting $\mathrm{ADs}$, where an alternative thrust coefficient $C_{\mathrm{T}}^{*}$ was used that is dependent on the local AD velocity, averaged over the $\mathrm{AD}:\left\langle U_{\mathrm{AD}}\right\rangle$. This avoids the necessity of a free-stream wind speed (which is not trivial for an AD operating in a wake) to look up the thrust coefficient. The $C_{\mathrm{T}}^{*}-\left\langle U_{\mathrm{AD}}\right\rangle$ curve was calculated from single RANS-AD simulations. In the present work, the AD control method is made wind speed independent by a simple additional scaling of $\left\langle U_{\mathrm{AD}}\right\rangle$, as explained in Sect. 2.2. This strategy is related to the work of Andersen (2014), where non-dimensional large-eddy simulations of wind turbines represented by actuator lines were coupled to an aero-elastic model that uses a dimensional controller. The extended AD control method can be utilized to perform one RANS-AD simulation to simulate the effect of different wind speeds without changing the inflow, thereby reducing the computational effort of a full AEP calculation by a factor of about 2-3 compared to using different inflow wind speeds.

\section{Methodology}

\subsection{Wind farm simulations}

The RANS-AD methodology used for our wind farm simulations are described in previous work (van der Laan et al., $2015 \mathrm{~b}$ ), and a brief summary is presented here. The RANS equation are solved in EllipSys3D, the in-house finite volume flow solver of DTU Wind Energy, initially developed by Sørensen (1994); Michelsen (1992). The $k-\varepsilon-f_{P}$ turbulence model is employed (van der Laan et al., 2015c), which has been developed for RANS-AD simulations using a neutral atmospheric surface layer. The 3-D flow domain represents a Cartesian domain with dimensions $\left(L_{x}=850 D\right.$, $L_{y}=830$ and $\left.L_{z}=10 D\right)$, for the streamwise $(x)$, lateral $(y)$ and vertical directions $(z)$, respectively. In the center of flow domain, a uniformly spaced domain is defined with dimensions $54 D \times 35 D \times 3 D$, in which the wind turbine wakes of $5 \times 5$ ADs are resolved. The ADs represent a wind farm of 25 National Renewable Energy Laboratory $5 \mathrm{MW}$ (NREL$5 \mathrm{MW}$ ) wind turbines (Jonkman et al., 2009) positioned in a rectangular layout with an interspacing of five rotor diameters $D$. The uniformly spaced domain around the wind farm has a cell size equal to $D / 8$, following a grid refinement study of previous work (van der Laan et al., 2015c). The cells sizes are grown while moving away from the wind farm. The grid consists of 352 blocks of $32^{3}$ cells, which adds up 11.5 million cells. One CPU per block is used in the simulations (352 CPUs in total). The boundaries at $x=0$ and $z=L_{z}$ are inlet conditions at which a logarithmic inflow profile for the streamwise velocity $U$, turbulent kinetic energy $k$ and dissipation of the turbulent kinetic energy $\varepsilon$ is set following Richards and Hoxey (1993). Periodic conditions are imposed on the lateral boundaries and an outflow boundary is set at $x=L_{x}$, at which all gradients in the streamwise direc- tion are assumed to be zero. The ground is set as a rough wall boundary condition following Sørensen et al. (2007). An offshore ambient turbulence intensity (based on $k$ ) of $6 \%$ is set at the wind turbine hub height of $90 \mathrm{~m}$ by using a (uniform) roughness length of $1.9 \times 10^{-4} \mathrm{~m}$. The convergence criterion in the RANS-AD simulations is set strict enough to calculate the AEP within a $0.02 \%$ convergence error.

\subsection{Wind-speed-independent AD control}

The new AD force control method is an extension of previous work (van der Laan et al., 2015a), where the total thrust on the $\mathrm{AD}$ is defined by $F_{\text {thrust }}=1 / 2 C_{\mathrm{T}}^{*} \rho A\left\langle U_{\mathrm{AD}}\right\rangle^{2}$. Here, $C_{\mathrm{T}}^{*}$ is the thrust coefficient based on the local AD velocity, averaged over the $\mathrm{AD}\left\langle U_{\mathrm{AD}}\right\rangle$, which can be calculated as $C_{\mathrm{T}}^{*}=\left(U_{\mathrm{H}} /\left\langle U_{\mathrm{AD}}\right\rangle\right)^{2}$, with $U_{\mathrm{H}}$ as the free-stream wind speed at hub height. The thrust force distribution of the AD is based on a normalized thrust force distribution computed by a detached eddy simulation of a blade-resolved NREL-5MW rotor for a below-rated wind speed of $8 \mathrm{~m} \mathrm{~s}^{-1}$ (Réthoré et al., 2014). The normalized thrust force distribution is scaled by $C_{\mathrm{T}}^{*}$ and $\left\langle U_{\mathrm{AD}}\right\rangle$. Hence, it is assumed that the thrust force distribution, based on a below-rated wind speed, does not change shape. This is generally not true for above-rated wind speeds, where the thrust force distribution is typically more uniform. However, Simisiroglou et al. (2017) has shown that the effect of different thrust force distributions (with constant total thrust force) on the velocity deficit is mainly visible in the near wake, while the far wake is almost unaffected, especially when atmospheric turbulence is present. In addition, the wake effects above rated wind speeds are small due to the low thrust coefficient. Hence, the effect of our assumed thrust force distribution on the annual energy production is expected to be small.

Prior to the wind farm simulations, a $C_{\mathrm{T}}^{*}-\left\langle U_{\mathrm{AD}}\right\rangle$ relation is calculated from a RANS-AD simulation of one AD for each wind speed between 4 and $25 \mathrm{~m} \mathrm{~s}^{-1}$, for every $1 \mathrm{~m} \mathrm{~s}^{-1}$, using the known $C_{\mathrm{T}}$ curve. We only use one RANS-AD simulation with a constant global inflow, where $C_{\mathrm{T}}$ is updated every time the simulation for a previous $C_{\mathrm{T}}$ has converged. The $C_{\mathrm{T}}^{*}-\left\langle U_{\mathrm{AD}}\right\rangle$ relation is used in the wind farm simulation to determine the thrust force for each iteration. The power is calculated from a $C_{\mathrm{P}}^{*}-\left\langle U_{\mathrm{AD}}\right\rangle$ relation, as a post-processing step, where $C_{\mathrm{P}}^{*}$ is defined as $C_{\mathrm{P}}^{*}=\left(U_{\mathrm{H}} /\left\langle U_{\mathrm{AD}}\right\rangle\right)^{3}$. If one would like include tangential forces to model wake rotation, it is possible to use a normalized tangential force distribution that is scaled by $C_{\mathrm{P}}^{*}$ and the tip speed ratio. In the present work, we do not use tangential forces, because their impact on the power deficit is very small (van der Laan et al., 2015b), and it allows us to use the symmetry of the chosen wind farm layout in order to reduce the number of wind directions necessary to calculate the AEP.

The precalculated $C_{\mathrm{T}}^{*}-\left\langle U_{\mathrm{AD}}\right\rangle$ used in this work is given in Fig. 1, where an additional scaling factor $s$ is added, which 


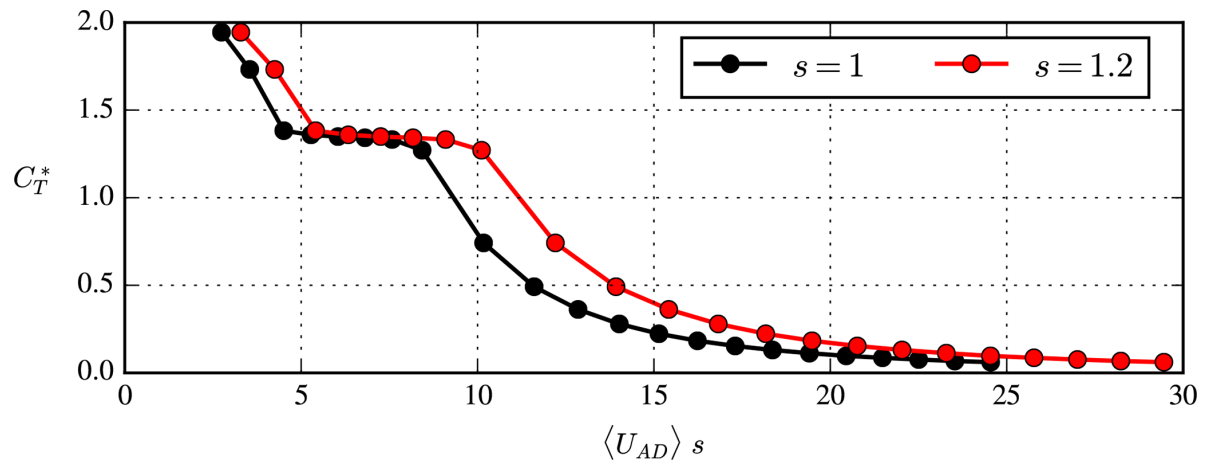

Figure 1. Precalculated $C_{\mathrm{T}}^{*}-\left\langle U_{\mathrm{AD}}\right\rangle$ curve used for $\mathrm{AD}$ control in wind farm simulations. Scaling parameter $s$ is used to represent different wind speeds.

is defined as

$s=U_{\mathrm{H}} / U_{\mathrm{H}, \text { inflow }}$,

where $U_{\mathrm{H}}$ is the free-stream wind speed at hub height that one would like to simulate and $U_{\mathrm{H} \text {,inflow }}$ is the actual inflow wind speed at hub height in the RANS-AD wind farm simulation. We multiply $\left\langle U_{\mathrm{AD}}\right\rangle$ in the $C_{\mathrm{T}}^{*}-\left\langle U_{\mathrm{AD}}\right\rangle$ by $s$. This simple scaling allows us to perform one RANS-AD wind farm simulation with a fixed inflow profile (e.g. $U_{\mathrm{H} \text {,inflow }}=10 \mathrm{~m} \mathrm{~s}^{-1}$ ) and use the scaling parameter $s$ to simulate different wind speeds $U_{\mathrm{H}}$ (e.g. $U_{\mathrm{H}}=12 \rightarrow s=1.2$ ). The control of the start and stop events of an AD is based on the 1-D momentum estimate of the free-stream velocity, as described in previous work (van der Laan and Abkar, 2019).

\section{Results and discussion}

The AEP of the $5 \times 5$ NREL-5MW wind farm is calculated with RANS-AD simulation(s) using 22 wind speeds between 4 and $25 \mathrm{~m} \mathrm{~s}^{-1}$ (every $1 \mathrm{~m} \mathrm{~s}^{-1}$ ) and 16 wind directions between 270 and $315^{\circ}$ (every $3^{\circ}$ ) using the symmetry of the wind farm layout. The wind farm layout is aligned with a wind direction of $270^{\circ}$. A baseline and five additional simulation methodologies are depicted in Fig. 2.

The baseline case represents 352 individual simulations, which need a total of $1.3 \times 10^{5}$ iterations on the finest grid $\left(i_{\text {tot }}\right)$. Each simulation is started with a twice-as-coarse grid (in all three directions) in order to reduce the number of required iterations on the finest grid. The coarse-grid CPU time is negligible compared to fine-grid CPU time, and the total number of iterations on the finest grid is representative of the computational effort. Without the multi-grid approach, one would need 2.4 times more iterations for the baseline case $\left(3.2 \times 10^{5}\right)$. The additional methods in Fig. 2 represent sequential or partly sequential simulation(s), where the next wind speed or wind direction is calculated from a previous result. This typically reduces the total number of required iterations because only local flow changes need to be simulated. However, the required number of iterations is directly proportional to the convergence of the local flow. Therefore, it will not be possible to speed up the computations further by using an initial guess based on, e.g. one of the fastengineering wake models. The first wind speed and wind direction for Methods I-V (filled circles in Fig. 2) is started from a coarser grid level similarly to the baseline case, while the following wind speed and wind direction are simulated on the finest grid only. The additional methods predict the same AEP within $0.02 \%$ of the baseline method, which is a result of the chosen convergence criterion. Method I represents 22 separate simulations (one for each wind speed), where the different wind directions are simulated sequentially after each other. The effect of wind direction is simulated by rotating the layout, while keeping the inflow constant. This is possible for RANS-AD simulations using flat terrain and a homogeneous roughness length. The total number of iterations of the finest grid for Method I is reduced by a factor $\left(f_{\text {red }}\right)$ of 2.2 compared to the baseline. In Method II, each wind direction is simulated individually, and the wind speeds are simulated sequentially using the proposed $\mathrm{AD}$ control method from Sect. 2.2, which reduces the number of fine-grid iterations by a factor 1.9. Methods III-V are each a single RAND-AD simulation, where all wind speeds and wind directions are simulated by rotation of the layout and scaling of the AD control, respectively. Methods III-V differ in the order of the simulated wind speeds and wind directions. In our presented methods, we choose to either change a wind speed or a wind direction with the smallest step but one could also choose alternative sequential solving paths. This is an optimization problem by itself and it is out of the scope of the present work. Methods III and IV provide similar reduction factors, although we have observed that there are differences in the required iterations for particular wind speed and wind direction flow cases. When the thrust coefficient in the wind farm is not varying much (for wind speeds between 7 and $\left.10 \mathrm{~m} \mathrm{~s}^{-1}\right)$ and well above rated $\left(>21 \mathrm{~m} \mathrm{~s}^{-1}\right.$ ), it takes less iterations to perform consecutive wind speeds with respect to consecutive wind directions. The opposite is observed for low wind speeds, when some of the wind tur- 

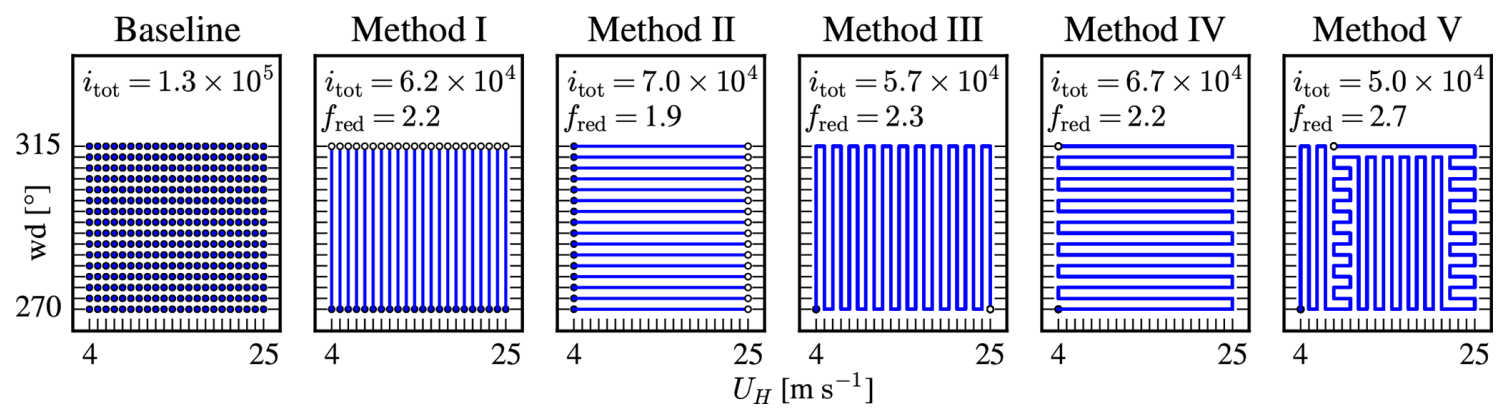

Figure 2. Parallel and sequential simulation methodologies to calculate the AEP using 22 wind speeds $\left(U_{\mathrm{H}}\right)$ and 16 wind directions (wd). Filled and open circles represent the start and end flow cases. $i_{\text {tot }}$ is the number of iterations on the finest grid level and $f_{\text {red }}$ is the reduction of these iterations compared to baseline case.

bines experience start-up and stop events (i.e. when going from 4 to $5 \mathrm{~m} \mathrm{~s}^{-1}$ or 5 to $4 \mathrm{~m} \mathrm{~s}^{-1}$ ) and where the thrust coefficient changes rapidly with wind speed (i.e. above the rated wind speed). Method V is a combination of Methods III and IV in order to further reduce the number of fine-grid iteration by a factor 2.7 compared to the baseline case. It should be noted that performance of Method $\mathrm{V}$ is dependent on the thrust coefficient curve and wind farm layout.

For RANS-AD simulations including terrain or inhomogeneous roughness lengths, it is not possible to rotate the layout while keeping the inflow direction constant. In this case, our proposed wind-speed-independent AD control method would reduce the number of required iterations and computational effort significantly following Method II. Note that RANS simulations of non-homogeneous terrain using a logarithmic inflow and rough wall boundary condition are also independent of the Reynolds number, for wind speeds that are relevant for wind turbines, as discussed by Troen et al. (2014). In addition, if one does not have a multi-grid available to reduce the number of fine-grid iterations compared to the baseline case, then the sequential simulation methods (Methods I-V, which do not benefit much from the multi-grid) yields an additional reduction factor of 2.4.

The total CPU time of the fastest method (Method V) is $1.4 \times 10^{3} \mathrm{CPU}$ hours, representing $4 \mathrm{~h}$ in wall-clock time. For non-symmetric wind farm layouts, one would need to perform more wind direction cases, i.e. 120 wind directions, which would increase the CPU hours and wall-clock time by a factor of about 7.5 representing a wall-clock time of about $30 \mathrm{~h}$ using 352 CPUs. By doubling the amount of CPUs, one could achieve an AEP calculation of our test wind farm within a day, which makes the RANS-AD model a feasible tool to validate the AEP of a wind farm designed by engineering wake models. It should be noted that if the user has unlimited computational resources available, then the baseline method is the fastest in terms of wall-clock time compared to Methods I-V because all wind speed and wind direction cases can be performed in parallel, which takes only a few minutes per simulation.
In the present work, we have simulated all wind speeds, also far above rated power where the wind turbines do not experience power losses. If one is only interested in the AEP, then these simulations could have been skipped once rated wind farm power is achieved. One could further reduce the computational effort of RANS-AD AEP calculations by reducing the number of wind speed and wind direction cases using statistics of the wind resources (i.e. using the Weibull and wind direction distributions), which could be investigated in future work.

Finally, these computations have been performed with an accuracy of $0.02 \%$ for the convergence error in AEP. One could choose to relax the convergence criteria of the solutions at the expense of an higher error but with a significant reduction in the total amount of computational iterations. For instance, one could choose to accept an error of $0.5 \%$, which would reduce the computational effort by a factor of 18 . An error of $0.5 \%$ would presumably still be less than the uncertainty associated with, for instance, the wind rose and $C_{\mathrm{T}}$ curve of the turbines. The freed computational resources could then be used to examine these larger uncertainties, and as the numerical error has been quantified, one could correct the final AEP assessment accordingly.

\section{Conclusions}

A simple wind-speed-independent actuator disk control method is proposed and employed to reduce the number of iterations necessary to calculate the annual energy production from Reynolds-averaged Navier-Stokes simulations of $5 \times 5$ rectangular wind farm with a spacing of five rotor diameters. The effects of different wind directions and wind speeds are calculated consecutively in a single simulation by rotating the wind farm layout and using the new wind-speed-independent actuator disk control method, respectively. Since the global inflow wind speed and wind direction are not changed, only local changes need to be recalculated for every wind speed and wind direction from a 
previous converged result, which reduces the total number of iterations by a factor of about $2-3$.

Code and data availability. The numerical results are generated with proprietary software, although the data presented can be made available by contacting the corresponding author. 
Appendix A: Reynolds number independence of a single wind turbine wake in RANS

The Reynolds number independence of the RANS-AD simulations for a single wind turbine wake is shown in Fig. A1, where the streamwise velocity $U$, normalized by the inflow wind speed at hub height, $U_{\mathrm{H} \text {,inflow }}$, is depicted at a downstream distance of five rotor diameters. The same numerical setup is used as discussed in Sect. 2, where we use a fixed force distribution scaled by $C_{\mathrm{T}}$ and $U_{\mathrm{H} \text {,inflow. We have simu- }}$ lated four different cases representing two thrust coefficients $\left(C_{\mathrm{T}}=0.4\right.$ and $\left.C_{\mathrm{T}}=0.8\right)$ and two ambient turbulence intensities at hub height $(I=5 \%$ and $I=10 \%)$ based on the turbulent kinetic energy. Each case is simulated with three different inflow wind speeds, namely, 1,10 and $100 \mathrm{~m} \mathrm{~s}^{-1}$, corresponding to Reynolds numbers of $8.7 \times 10^{6}, 8.7 \times 10^{7}$ and $8.7 \times 10^{8}$, respectively, for the chosen kinematic molecular viscosity of $1.46 \times 10^{-5} \mathrm{~m}^{2} \mathrm{~s}^{-1}$. Figure A1 clearly shows that normalized velocity deficit is independent of the wind speed.

\begin{tabular}{|c|c|c|c|}
\hline$I=5 \%, C_{T}=0.4:$ & $-\infty U_{H, \text { inflow }}=1 \mathrm{~ms}^{-1}$ & $-U_{H, \text { inflow }}=10 \mathrm{~ms}^{-1}$ & $\rightarrow U_{H, \text { inflow }}=100 \mathrm{~ms}^{-1}$ \\
\hline$I=5 \%, C_{T}=0.8:$ & $-\infty U_{H, \text { inflow }}=1 \mathrm{~ms}^{-1}$ & $-U_{H, \text { inflow }}=10 \mathrm{~ms}^{-1}$ & $\rightarrow U_{H, \text { inflow }}=100 \mathrm{~ms}^{-1}$ \\
\hline$I=10 \%, C_{T}=0.4:$ & $\multimap U_{H, \text { inflow }}=1 \mathrm{~ms}^{-1}$ & $-U_{H, \text { inflow }}=10 \mathrm{~ms}^{-1}$ & $\rightarrow U_{H, \text { inflow }}=100 \mathrm{~ms}^{-1}$ \\
\hline$I=10 \%, C_{T}=0.8:$ & $-U_{H, \text { inflow }}=1 \mathrm{~ms}^{-1}$ & $-U_{H, \text { inflow }}=10 \mathrm{~ms}^{-1}$ & $\rightarrow U_{H, \text { inflow }}=100 \mathrm{~ms}^{-1}$ \\
\hline
\end{tabular}
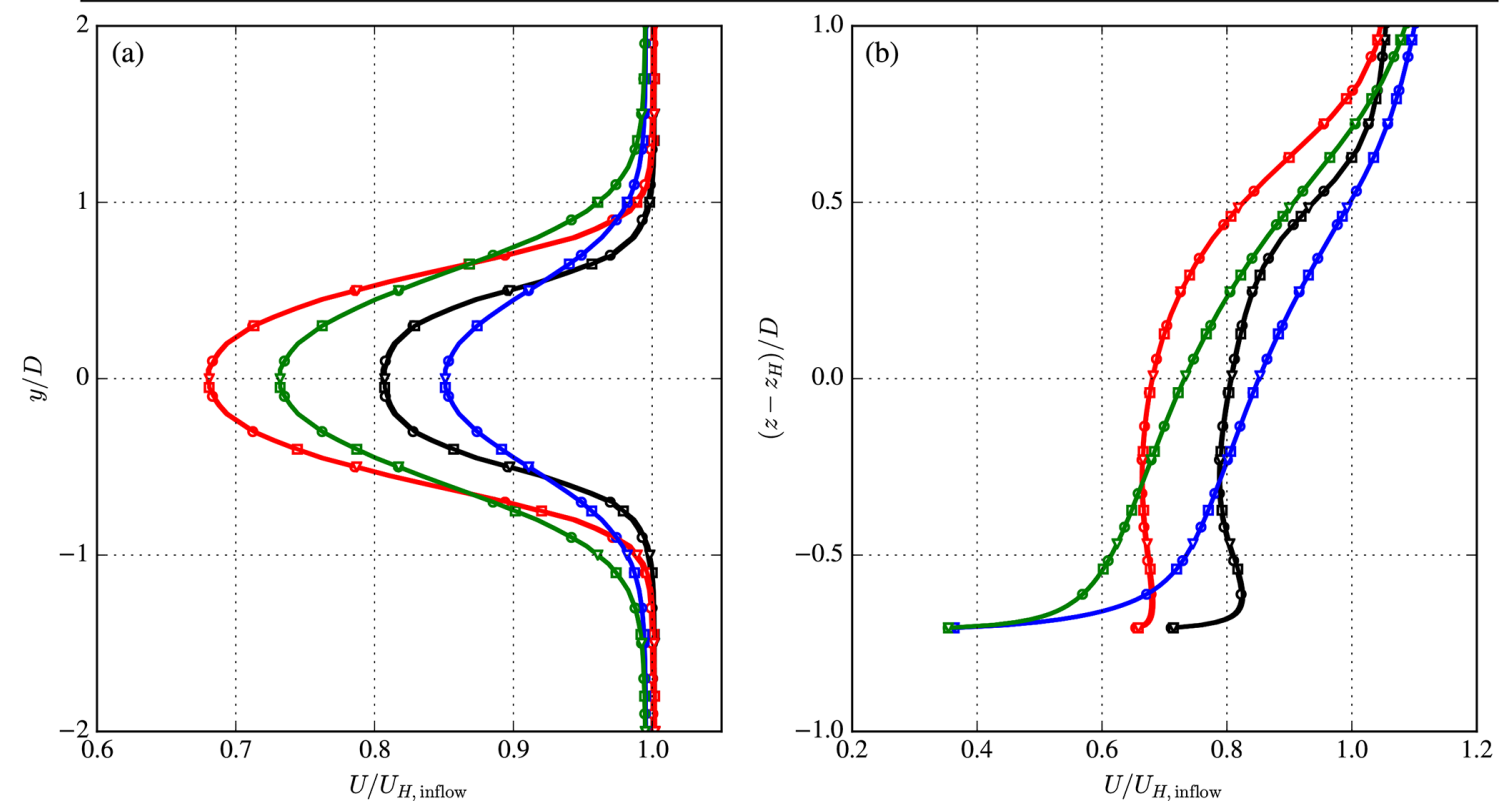

Figure A1. Reynolds number independence of the streamwise velocity deficit of a single $\mathrm{AD}$ with a prescribed thrust coefficient $C_{\mathrm{T}}$ and ambient turbulence intensity $I$, at downstream distance of five rotor diameters. (a) Streamwise velocity at hub height as function of the lateral coordinate. (b) Streamwise velocity at $y=0$ as function of the vertical coordinate. 
Author contributions. MPVDL performed the simulations, produced all figures and drafted the article. SJA and P-ER contributed to the methodology and finalization of the paper

Competing interests. The authors declare that they have no conflict of interest.

Review statement. This paper was edited by Carlo L. Bottasso and reviewed by two anonymous referees.

\section{References}

Andersen, S.: Simulation and Prediction of Wakes and Wake Interaction in Wind Farms, PhD thesis, Wind Energy Department, Technical University of Denmark, 2014.

Barthelmie, R. J., Frandsen, S. T., Nielsen, M. N., Pryor, S. C., Rethore, P. E., and Jørgensen, H. E.: Modelling and measurements of power losses and turbulence intensity in wind turbine wakes at middelgrunden offshore wind farm, Wind Energy, 10, 517-528, https://doi.org/10.1002/we.238, 2007.

Göçmen, T., van der Laan, M. P., Réthoré, P. E., Peña Diaz, A., Larsen, G. C., and Ott, S.: Wind turbine wake models developed at the technical university of Denmark: A review, Renewable and Sustainable Energy Reviews, 60, 752-769, 2016.

Jonkman, J., Butterfield, S., Musial, W., and Scott, G.: Definition of a 5-MW Reference Wind Turbine for Offshore System Development, Tech. rep., National Renewable Energy Laboratory, 2009.

Michelsen, J. A.: Basis3D - a platform for development of multiblock PDE solvers., Tech. Rep. AFM 92-05, Technical University of Denmark, Lyngby, Denmark, 1992.

Réthoré, P.-E., van der Laan, M. P., Troldborg, N., Zahle, F., and Sørensen, N. N.: Verification and validation of an actuator disc model, Wind Energy, 17, 919-937, 2014.
Richards, P. J. and Hoxey, R. P.: Appropriate boundary conditions for computational wind engineering models using the $k-\varepsilon$ turbulence model, J. Wind Eng. Ind. Aerod., 46,47, 145-153, 1993.

Simisiroglou, N., Breton, S.-P., and Ivanell, S.: Validation of the actuator disc approach using small-scale model wind turbines, Wind Energ. Sci., 2, 587-601, https://doi.org/10.5194/wes-2587-2017, 2017.

Sørensen, N. N.: General purpose flow solver applied to flow over hills, PhD thesis, Risø National Laboratory, Roskilde, Denmark, 1994.

Sørensen, N. N., Bechmann, A., Johansen, J., Myllerup, L., Botha, P., Vinther, S., and Nielsen, B. S.: Identification of severe wind conditions using a Reynolds Averaged Navier-Stokes solver, J. Phys. Conf. Ser., 75, 1-13, https://doi.org/10.1088/17426596/75/1/012053, 2007

Troen, I., Bechmann, A., Kelly, M. C., Sørensen, N. N., Réthoré, P.-E., Cavar, D., and Jørgensen, H. E.: Complex terrain wind resource estimation with the wind-atlas method: Prediction errors using linearized and nonlinear CFD micro-scale models, in: Proceedings of European Wind Energy Association, 2014.

van der Laan, M. P. and Abkar, M.: Improved energy production of multi-rotor wind farms, J. Phys. Conf. Ser., https://doi.org/10.1088/1742-6596/1256/1/012011, 2019.

van der Laan, M. P., Sørensen, N. N., Réthoré, P.-E., Mann, J., Kelly, M. C., and Troldborg, N.: The $k-\varepsilon-f_{P}$ model applied to double wind turbine wakes using different actuator disk force methods, Wind Energy, 18, 2223-2240, https://doi.org/10.1002/we.1816, 2015a.

van der Laan, M. P., Sørensen, N. N., Réthoré, P.-E., Mann, J., Kelly, M. C., Troldborg, N., Hansen, K. S., and Murcia, J. P.: The $k-\varepsilon-$ $f_{P}$ model applied to wind farms, Wind Energy, 18, 2065-2084, https://doi.org/10.1002/we.1804, 2015 b.

van der Laan, M. P., Sørensen, N. N., Réthoré, P.-E., Mann, J., Kelly, M. C., Troldborg, N., Schepers, J. G., and Machefaux, E.: An improved $k-\varepsilon$ model applied to a wind turbine wake in atmospheric turbulence, Wind Energy, 18, 889-907, https://doi.org/10.1002/we.1736, 2015c. 